\title{
Research Article \\ On the Geolocation Bounds for Round-Trip Time-of-Arrival and All Non-Line-of-Sight Channels
}

\author{
Laurence Mailaender \\ Alcatel-Lucent, Bell Labs, Holmdel, NJ 07733, USA \\ Correspondence should be addressed to Laurence Mailaender, lm@alcatel-lucent.com
}

Received 20 February 2007; Revised 31 July 2007; Accepted 28 October 2007

Recommended by Richard J. Barton

The development of future geolocation systems requires a fundamental understanding of the importance of various system parameters, such as the number of sensors, the SNR, bandwidth, and channel conditions. We consider the bounds on time-based geolocation accuracy when all sensors experience non-line-of-sight (NLOS) conditions. While location accuracy generally improves with additional bandwidth, we find that NLOS effects place a limit on these gains. Our evaluation focuses on indoor geolocation where Rayleigh fading is present, different average SNR conditions occur on each link, and the sensors may not fully encircle the user. We introduce a new bound for round-trip time-of-arrival (RT-TOA) systems. We find that time-of-arrival (TOA) outperforms time-difference-of-arrival TDOA and RT-TOA, but the relative ordering of the latter two depends on the sensor geometry.

Copyright ( $\odot 2008$ Laurence Mailaender. This is an open access article distributed under the Creative Commons Attribution License, which permits unrestricted use, distribution, and reproduction in any medium, provided the original work is properly cited.

\section{INTRODUCTION}

The success of the global positioning system (GPS) has sparked great interest in locating mobile wireless users. Applications include locating E-911 callers in the cellular telephone network, tracking emergency responder personnel in indoor environments, personal navigation using maps and turn-by-turn driving directions, or tracking vehicles and other valuable property. All such systems will require improvements in the delivered accuracy, time-to-fix, reduction of outage probability, and lowered cost. We can also foresee a shift from a global system to positioning based on local infrastructure, possibly relying entirely on transmissions from ground stations. With a local architecture comes the possibility of combining wideband communications and location functions in the same radio spectrum for increased spectrum utilization.

To enable the design of these future systems, there is a continuing need to understand the fundamental performance limits of positioning systems, especially in difficult indoor and urban environments [1-4]. While at first glance it may appear that simply increasing the signaling bandwidth will lead to gains in location accuracy, several effects emerge that limit such gains. First, a larger bandwidth implies an in- crease in the observed multipath delay spread (in samples), making the detection of the "first arrival" signal more difficult in practice. Next, urban/indoor environments also exhibit a "non-line-of-sight" (NLOS) property (also known as "undetectable direct path"), wherein the path due to straightline propagation may be severely attenuated and considered undetectable. This is a substantial obstacle to accurate localization. Several authors have proposed algorithms for locating users in either pure NLOS or mixed LOS/NLOS conditions [5-8]. A statistical characterization of the NLOS effect is not presently available, though a few authors have recently published results in this direction $[9,10]$. Also needed is an understanding of the performance differences in these environments among several positioning principles: timeof-arrival (TOA), time-difference-of-arrival (TDOA), and round-trip time-of-arrival (RT-TOA).

Our focus in this paper is on the theoretical performance limit, given by the Cramer-Rao lower bound (CRLB), for the three time-based location principles when all the sensors exhibit NLOS conditions. If the total number of sensors is small, it may not be feasible to separate out the LOS sensors and perform the location fix from that subset only; hence all sensors should be considered NLOS to some degree. We envision indoor location using only ground-based infrastructure, 
which brings the advantages of lowered Doppler, reduced delay uncertainty, higher signal strength, and lack of ionospheric effects. We also assume Rayleigh fading of the signals (implying that the Cramer-Rao bound becomes a random variable) and different average SNR at each sensor due to pathloss and shadowing. Two other assumptions should be mentioned up front. We will restrict ourselves to the case of flat fading only, as it has been shown [3] that multipath actually improves the CRLB slightly (theoretically, it is not a degradation), while introducing numerical complications. Also, certain advanced location receivers, using integrated carrier phase $[11,12]$, are able to achieve extraordinary location accuracy by examining the carrier signal phase prior to mixing to baseband. These systems currently have severe limitations in terms of the time-to-fix, and the allowed initial position uncertainty, so we restrict ourselves to traditional baseband processing.

The unique contributions of this paper are as follows. We present the first (to the author's knowledge) derivation of the CRLB for a RT-TOA system in LOS and NLOS channels, and provide numerical comparisons with TOA and TDOA. We investigate the CRLB under pure NLOS channels and prove that the bound does not exist when there is no a priori information. We prove that a priori channel amplitude information is not needed to achieve the CRLB. We also investigate the CRLB when the sensor's locations are themselves only statistically known, again, for the first time to our knowledge under pure NLOS channels. Additionally, two variants of RT-TOA are investigated, one of which is shown to have root-mean-square (rms) error precisely twice that of TOA.

In Section 2, we begin with a review of the Cramer-Rao bound, and the location bounds for TOA and TDOA processing. The need for a priori amplitude information is addressed. In Section 3, we look at the all-NLOS case, and introduce a "half Gaussian" model for the probability density function (pdf) of the excess delay. In Section 4 the RT-TOA system is investigated, and in Section 5, we study statistical sensor position knowledge. Finally, Section 6 presents our numerical findings, and Section 7 contains a summary and conclusions.

\section{CRAMER-RAO BOUNDS FOR GEOLOCATION}

The Cramer-Rao lower bound (CRLB) gives the lower limit on estimation error among all unbiased estimators. Consider a parameter vector $\boldsymbol{\theta}=\left[x, y, p_{1}, \ldots, p_{N}\right]^{T}$ made up of the parameters of direct interest, here, the two-dimensional position ${ }^{1}$ of the terminal, $[x, y]$, and additional unknown nuisance parameters $\left[p_{1}, \ldots, p_{N}\right]$ that affect the received vector, r. The Fisher matrix [13] is

$$
\mathbf{J}_{\theta}=E\left\{\left(\frac{\delta}{\delta \boldsymbol{\theta}} \Lambda(\mathbf{r} \mid \boldsymbol{\theta})\right)\left(\frac{\delta}{\delta \boldsymbol{\theta}} \Lambda(\mathbf{r} \mid \boldsymbol{\theta})\right)^{H}\right\}
$$

\footnotetext{
${ }^{1}$ Extension of all results to the three-dimensional case is straight-forward.
}

where $\Lambda(\mathbf{r} \mid \boldsymbol{\theta})$ is the log-likelihood of received vector, $\mathbf{r}$. Note that the expectation in (1) is over the random variable, $\mathbf{r}$. The Cramer-Rao lower bound for the position error is then

$$
\mathrm{CRLB}=\operatorname{Tr}\left\{\left(\mathbf{J}_{\theta}^{-1}\right)_{2 \times 2}\right\},
$$

where the notation indicates taking the trace of the upperleft corner of the inverse of the full Fisher matrix. The rms position error is the square root of this value.

It is also possible that some of the variables in the parameter vector are partially known, meaning that we know their a priori probability density, $p(\boldsymbol{\omega})$. In this case, it can be shown that the Fisher matrix is

$$
\mathbf{J}=\mathbf{J}_{\theta}+\mathbf{J}_{\omega}
$$

where

$$
\mathbf{J}_{\omega}=E\left\{\left(\frac{\delta}{\delta \boldsymbol{\omega}} \Lambda(\boldsymbol{\omega})\right)\left(\frac{\delta}{\delta \boldsymbol{\omega}} \Lambda(\boldsymbol{\omega})\right)^{H}\right\}
$$

Here the expectation is over the random variable $\boldsymbol{\omega}$, and $\Lambda(\boldsymbol{\omega}) \triangleq \ln p(\boldsymbol{\omega})$. The generalized Cramer-Rao bound [14] is

$$
\mathrm{G}-\mathrm{CRLB}=\operatorname{Tr}\left\{\left(\mathbf{J}^{-1}\right)_{2 \times 2}\right\}
$$

\subsection{One-dimensional ranging bound}

Next we briefly review the derivation of the well-known Cramer-Rao bound for the one-dimensional ranging, or time-delay estimation problem. Here the channel conditions are assumed to be the ordinary LOS case, and the receiver is subject to ordinary AWGN. Assume a known waveform, $s(t)$, has been transmitted, and the received signal over a finite time interval is

$$
r(t)=s(t-\tau)+n(t), \quad 0 \leq t \leq T .
$$

The parameter of interest is the delay of the received signal, $\tau$, which is proportional to the distance between the receiver and transmitter. The ranging bound is derived from continuous-time estimation theory. The log-likelihood of a continuous-time random process is given by the CameronMartin formula [15]

$$
\Lambda(r \mid \tau)=\frac{2}{N_{0}} \int_{0}^{T} s(t-\tau) d X-\frac{1}{N_{0}} \int_{0}^{T} s(t-\tau)^{2} d t,
$$

where $X$ refers to the integral of the observed Wiener process. Using (1), the Fisher matrix (here, a scalar) for this problem then simplifies to

$$
J_{\tau}=\frac{2}{N_{0}} \int_{0}^{T}\left(\frac{\delta}{\delta \tau} s(t-\tau)\right)^{2} d t
$$

Letting $T \rightarrow \infty$ and assuming finite energy signals, we can use well-known Fourier transform properties. For the transmitted signal in (6), assume the Fourier pair $s(t) \Leftrightarrow S(f)$, 
and note that the transform of the time derivative is $\dot{s}(t) \Leftrightarrow$ $j 2 \pi f S(f)$. Applying Parseval's theorem, we find

$$
J_{\tau}=\frac{2}{N_{0}} \int_{0}^{\infty}(\dot{s}(t))^{2} d t=\frac{2}{N_{0}}(2 \pi)^{2} \int_{-\infty}^{\infty} f^{2}|S(f)|^{2} d f .
$$

Defining the squared rms bandwidth and the received signal energy as

$$
\begin{gathered}
\beta^{2} \triangleq \frac{\int_{-\infty}^{\infty} f^{2}|S(f)|^{2} d f}{\int_{-\infty}^{\infty}|S(f)|^{2} d f} \\
E_{\mathrm{obs}}=\int_{0}^{\infty}|s(t)|^{2} d t=\frac{1}{2 \pi} \int_{-\infty}^{\infty}|S(f)|^{2} d f \triangleq C T_{\mathrm{obs}},
\end{gathered}
$$

then substituting in and inverting (9) allow us to write the Cramer-Rao bound for ranging accuracy:

$$
\sigma_{\text {range }} \geq \frac{c}{\sqrt{2} 2 \pi \beta \sqrt{\mathrm{SNR}}},
$$

where $c$ is the speed of light. Note that ranging accuracy improves linearly with rms bandwidth, but only as the square root of SNR. Here we define SNR $\triangleq E_{\text {obs }} / N_{0}=\left(C / N_{0}\right) T_{\text {obs}}$. Note that observation interval $T_{\mathrm{obs}}$ is a function of the channel coherence time and the local oscillator stability, and not the signaling bandwidth. The implication is that if a transmitter has $C$ watts of average power, and we change the signaling bandwidth, then SNR as defined does not change. This fact will be important in the subsequent development.

\subsection{Basic geolocation bounds}

When the mobile user's and sensors' clocks are all synchronized, it is possible to use the TOA location technique. In the simplest form of the problem, no nuisance variables are considered, and the parameter vector is $\boldsymbol{\theta}=[x, y]$. The signal received at the $i$ th sensor is $r_{i}(t)=a_{i} s\left(t-\tau_{i}\right)+n_{i}(t)$, where $a_{i}$ is a complex-valued channel coefficient (here assumed known), and $\tau_{i}$ is the delay between the $i$ th sensor and the mobile user. Note that

$$
\tau_{i}=\frac{1}{c} \sqrt{\left(x_{i}-x\right)^{2}+\left(y_{i}-y\right)^{2}}
$$

where $\left(x_{i}, y_{i}\right)$ is the location of the $i$ th sensor. Using a vector derivative version of (8) and assuming $B$ sensors, the Fisher matrix is

$$
\mathbf{J}_{\theta}=\frac{2}{N_{o}} \sum_{i=1}^{B} \int_{0}^{T}\left[\frac{\delta}{\delta \boldsymbol{\theta}} a_{i} s\left(t-\tau_{i}\right)\right]\left[\frac{\delta}{\delta \boldsymbol{\theta}} a_{i} s\left(t-\tau_{i}\right)\right]^{H} d t .
$$

From elementary calculus,

$$
\begin{array}{rl}
\frac{\delta}{\delta \boldsymbol{\theta}} a_{i} & s\left(t-\frac{1}{c} \sqrt{\left(x_{i}-x\right)^{2}+\left(y_{i}-y\right)^{2}}\right) \\
& =\frac{a_{i}}{c} \dot{s}\left(t-\tau_{i}\right)\left[\frac{x_{i}-x}{\sqrt{\left(x_{i}-x\right)^{2}+\left(y_{i}-y\right)^{2}}}\right. \\
\triangleq & \left.\frac{y_{i}-y}{\sqrt{\left(x_{i}-x\right)^{2}+\left(y_{i}-y\right)^{2}}}\right] \\
& \dot{s}\left(t-\tau_{i}\right) \mathbf{h}_{i},
\end{array}
$$

where $\dot{s}(t)$ again denotes the time derivative of the transmitted signal. The Fisher matrix for TOA is

$$
\mathbf{J}_{\theta}=\sum_{i=1}^{B} \frac{2 E_{\mathrm{obs}, i}}{N_{o}}(2 \pi)^{2} \beta^{2} \frac{\left|a_{i}\right|^{2}}{c^{2}} \mathbf{h}_{i} \mathbf{h}_{i}^{H}=\mu_{1} \mathbf{H} \mathbf{\Lambda} \mathbf{H}^{H},
$$

where $\mathbf{H} \triangleq\left[\mathbf{h}_{1}, \mathbf{h}_{2}, \ldots, \mathbf{h}_{B}\right], \boldsymbol{\Lambda}$ is a diagonal matrix whose $i$ th element is $\left(E_{\mathrm{obs}, i} / N_{0}\right)\left|a_{i}\right|^{2}$ and $\mu_{1} \triangleq 2(2 \pi)^{2} \beta^{2} / c^{2}$. The CRLB therefore depends on the SNR per sensor link, the rms signal bandwidth, and the geometry of the user and sensors, and is computed from (2).

When the mobile user's clock is not synchronized with the sensors, we may use the TDOA location method. The most straightforward way of finding the related bound is by introducing a single nuisance variable, $\Delta_{0}$, that corresponds to the erroneous range due to clock offset. Hence, the parameter vector becomes $\boldsymbol{\theta}=\left[x, y, \Delta_{0}\right]$. It has already been shown that this very general approach is equivalent to the CRLB when delays are first estimated by ML and then one sensor's delay is subtracted from all the remaining delay estimates, corresponding to the classical TDOA processing [4]. We now write

$$
\tau_{i}=\frac{1}{c}\left(\sqrt{\left(x_{i}-x\right)^{2}+\left(y_{i}-y\right)^{2}}+\Delta_{0}\right)
$$

accounting for the unknown clock offset affecting each measurement. Starting from (13), we observe

$$
\begin{aligned}
& \frac{\delta}{\delta \boldsymbol{\theta}} a_{i} s\left(t-\frac{\sqrt{\left(x_{i}-x\right)^{2}+\left(y_{i}-y\right)^{2}}-\Delta_{0}}{c}\right) \\
& =\frac{a_{i}}{c} \dot{s}\left(t-\tau_{i}\right)\left[\begin{array}{c}
\frac{x_{i}-x}{\sqrt{\left(x_{i}-x\right)^{2}+\left(y_{i}-y\right)^{2}}} \\
\frac{y_{i}-y}{\sqrt{\left(x_{i}-x\right)^{2}+\left(y_{i}-y\right)^{2}}} \\
-1
\end{array}\right] \\
& \triangleq \frac{a_{i}}{c} \dot{s}\left(t-\tau_{i}\right) \overline{\mathbf{h}}_{i} \text {. }
\end{aligned}
$$

The Fisher matrix for the TDOA system is $\mu_{1} \overline{\mathbf{H}} \Lambda \overline{\mathbf{H}}^{H}$, where $\overline{\mathbf{H}} \triangleq\left[\overline{\mathbf{h}}_{1}, \overline{\mathbf{h}}_{2}, \ldots, \overline{\mathbf{h}}_{B}\right]$, and the CRLB is from (2). In our numerical results we will contrast the performance of TOA and TDOA systems. It has previously been shown that the performance of TDOA is no better than TOA, and equality is proven to occur only in certain highly symmetrical sensor geometries [2].

\subsection{CRLB with unknown signal amplitudes}

Thus far, we have considered the CRLB when the signal amplitudes were assumed known. In this section, we redefine the parameter vector to be $\boldsymbol{\theta}=\left[x, y, a_{1}, \ldots, a_{N}\right]^{T}$ and consider the impact of these unknown nuisance variables. Note 
that [1] considered a similar case of joint amplitude/delay channel estimation. In our case,

$$
\begin{gathered}
\frac{\delta}{\delta \boldsymbol{\theta}} a_{i} s\left(t-\frac{1}{c} \sqrt{\left(x_{i}-x\right)^{2}+\left(y_{i}-y\right)^{2}}\right) \\
=\left[\begin{array}{c}
a_{i} \dot{s}\left(t-\tau_{i}\right) \frac{\left(x_{i}-x\right)}{c \sqrt{\left(x_{i}-x\right)^{2}+\left(y_{i}-y\right)^{2}}} \\
a_{i} \dot{s}\left(t-\tau_{i}\right) \frac{\left(y_{i}-y\right)}{c \sqrt{\left(x_{i}-x\right)^{2}+\left(y_{i}-y\right)^{2}}} \\
\mathbf{0}
\end{array}\right]_{(B+2) \times 1}\left(t-\tau_{i}\right) \\
\mathbf{0}
\end{gathered},
$$

where it should be understood that the first two terms are generally nonzero for all $i$, and the remaining nonzero term occurs in the $(i+2)$ th position. Define $\mu_{2} \triangleq \int_{0}^{T} s(t) \dot{s}(t) d t=$ $0.5\left(s^{2}(T)-s^{2}(0)\right)$. The Fisher matrix is

$$
\mathbf{J}_{\theta}=\left[\begin{array}{cc}
\mu_{1} \mathbf{H} \mathbf{\Lambda} \mathbf{H}^{H} & \mu_{2} \mathbf{H} \boldsymbol{\Lambda}^{1 / 2} \\
\mu_{2} \Lambda^{H / 2} \mathbf{H}^{H} & \mathbf{D}
\end{array}\right],
$$

where $\mathbf{D} \triangleq \operatorname{diag}\left(2 E_{\mathrm{obs}, 1} / N_{0}, \ldots, 2 E_{\mathrm{obs}, B} / N_{0}\right)$. Given this structure, we would like to know if the unknown amplitudes affect the bound, or whether

$$
\operatorname{Tr}\left(\left(\mathbf{J}_{\theta}^{-1}\right)_{2 \times 2}\right) \triangleq \operatorname{Tr}\left(\left(\mu_{1} \mathbf{H} \mathbf{\Lambda} \mathbf{H}^{H}\right)^{-1}\right) .
$$

This question is especially relevant in the multipath channel case, where the unknown amplitudes would include the complete time-dispersive channel structure, but we do not consider this here.

Theorem 1. The degradation of the CRLB due to unknown channel amplitudes can be made arbitrarily small.

Proof. The key observation is that the scalar $\mu_{2}$ is under the control of the designer, and may be made arbitrarily small. When $\mu_{2}=0$, then $\mathbf{J}_{\theta}$ of (19) becomes block diagonal, and (20) follows immediately.

Due to this result, the unknown amplitude case will not be considered further.

\section{GEOLOCATION WITH ALL NLOS SENSORS}

In [1-4] the sensors experienced a mix of LOS and NLOS conditions and the receiver was assumed to have perfect knowledge which were LOS. Here, we consider all sensors to experience NLOS channels. Let $N_{i}$ be the unknown, additional range the signal travels between the user and the $i$ th sensor. The corresponding signal delay is

$$
\tau_{i}=\frac{1}{c}\left(\sqrt{\left(x_{i}-x\right)^{2}+\left(y_{i}-y\right)^{2}}+N_{i}\right) .
$$

We expand the parameter vector to include these unknown nuisance variables, $\boldsymbol{\theta}=\left[x, y, N_{1}, \ldots, N_{B}\right]^{T}$. Taking derivatives as in (14) we find the Fisher matrix in this case to be

$$
\mathbf{J}_{\theta}=\sum_{i=1}^{B} \frac{2 E_{\mathrm{obs}, i}}{N_{0}}(2 \pi)^{2} \beta^{2} \frac{\left|a_{i}\right|^{2}}{c^{2}}\left[\begin{array}{c}
\mathbf{h}_{i} \\
-\mathbf{e}_{i}
\end{array}\right]\left[\begin{array}{c}
\mathbf{h}_{i} \\
-\mathbf{e}_{i}
\end{array}\right]^{H},
$$

where $\mathbf{e}_{i}$ is the elementary column vector of length $B$, having a single 1 in the $i$ th position. The Fisher matrix is of size $(B+$ $2) \times(B+2)$ and can also be written as

$$
\mathbf{J}_{\theta}=\mu_{1}\left[\begin{array}{cc}
\mathbf{H} \mathbf{\Lambda} \mathbf{H}^{H} & -\mathbf{H} \boldsymbol{\Lambda} \\
-\boldsymbol{\Lambda} \mathbf{H}^{H} & \boldsymbol{\Lambda}
\end{array}\right]
$$

where $\mathbf{H}$ and $\boldsymbol{\Lambda}$ are as defined previously. However, in the all-NLOS case, the inverse of this Fisher matrix does not exist, implying that there can be no guarantee of a finite error variance.

Theorem 2. When a TOA or a TDOA geolocation system experiences NLOS conditions on all sensors and lacks a priori information, the resulting Fisher matrix is singular, and the Cramer-Rao bound does not exist.

Proof. Consider first a TOA system. Assume that the CRLB exists in the related LOS conditions, that is, $\left(\mathbf{H} \mathbf{\Lambda} \mathbf{H}^{H}\right)^{-1}$ exists. Regarding (23), the inverse will exist iff the determinant is nonzero. The determinant is

$$
\begin{aligned}
\left|\mathbf{J}_{\theta}\right| & =\mu_{1}^{B}\left|\mathbf{H} \mathbf{\Lambda} \mathbf{H}^{H}\right|\left|\boldsymbol{\Lambda}-\mathbf{\Lambda} \mathbf{H}^{H}\left(\mathbf{H} \mathbf{\Lambda} \mathbf{H}^{H}\right) \mathbf{H} \mathbf{\Lambda}\right| \\
& =\mu_{1}^{B}\left|\mathbf{H} \mathbf{\Lambda} \mathbf{H}^{H}\right||\mathbf{S}| .
\end{aligned}
$$

The first determinant is nonzero by assumption. The second determinant is nonzero if and only if $\mathbf{S}^{-1}$ exists. Using the matrix inversion lemma,

$$
\begin{aligned}
\mathbf{S}^{-1} & =\boldsymbol{\Lambda}^{-1}-\boldsymbol{\Lambda}^{-1} \boldsymbol{\Lambda} \mathbf{H}^{H}\left(\mathbf{H} \mathbf{\Lambda} \boldsymbol{\Lambda}^{-1} \boldsymbol{\Lambda} \mathbf{H}^{H}-\mathbf{H} \mathbf{\Lambda} \mathbf{H}^{H}\right)^{-1} \mathbf{H} \mathbf{\Lambda} \mathbf{\Lambda}^{-1} \\
& =\boldsymbol{\Lambda}^{-1}-\mathbf{H}^{H}\left(\mathbf{H} \boldsymbol{\Lambda} \mathbf{H}^{H}-\mathbf{H} \boldsymbol{\Lambda} \mathbf{H}^{H}\right)^{-1} \mathbf{H},
\end{aligned}
$$

where the second term clearly does not exist. Therefore, $\left(\mathbf{J}_{\theta}\right)^{-1}$ does not exist. The same proof extends immediately to the TDOA case when $\overline{\mathbf{H}}$ is substituted for $\mathbf{H}$, and assuming $\left(\overline{\mathbf{H}} \Lambda \overline{\mathbf{H}}^{H}\right)^{-1}$ exists.

\subsection{Extension when the statistics of the NLOS are known}

Consider again the parameter vector $\boldsymbol{\theta}=\left[x, y, N_{1}, \ldots, N_{B}\right]^{T}$ and the delays defined in (21). When the probability density function $p_{N}(\mathbf{n})$ is known, then we can write the Fisher matrix using (3):

$$
\mathbf{J}=\mu_{1}\left[\begin{array}{cc}
\mathbf{H} \boldsymbol{\Lambda} \mathbf{H}^{H} & -\mathbf{H} \boldsymbol{\Lambda} \\
-\boldsymbol{\Lambda} \mathbf{H}^{H} & \boldsymbol{\Lambda}
\end{array}\right]+\left[\begin{array}{cc}
\mathbf{0}_{2 \times 2} & \mathbf{0}_{2 \times B} \\
\mathbf{0}_{B \times 2} & \boldsymbol{\Omega}_{B \times B}
\end{array}\right],
$$

where the subscripts denote the dimensions of the various submatrices. We define $\mathbf{n} \triangleq\left[N_{1}, N_{2}, \ldots, N_{B}\right]^{T}$, and

$$
\boldsymbol{\Omega}=E\left\{\left[\frac{\partial}{\partial \mathbf{n}} \ln p_{N}(\mathbf{n})\right]\left[\frac{\partial}{\partial \mathbf{n}} \ln p_{N}(\mathbf{n})\right]^{H}\right\},
$$


where the expectation is over $\mathbf{n}$. Note that the choice of density $p_{N}(\mathbf{n})$ affects whether (26) is invertible. At the present time, very little is known about the statistics of the NLOS variables in realistic propagation environments, and there are no established models. In [1] the numerical results assumed an ordinary two-sided Gaussian distribution which includes negative delays. Since the straight-line path is the shortest path, any other NLOS path can only increase the delay, so it would be more reasonable to use a density having support only on $\mathbf{n} \geq 0$. As an example, we might consider use of the exponential distribution,

$$
p_{N}(\mathbf{n})=\prod_{n=1}^{N} \frac{1}{\sigma} e^{-n_{i} / \sigma}, \quad n_{i} \geq 0
$$

This is attractive as it has support only for positive delays and is easy to differentiate. Substituting (28) into (27),

$$
\ln \left(p_{N}(\mathbf{n})\right)=\sum_{i=1}^{B} \ln \left(\frac{1}{\sigma} e^{-n_{i} / \sigma}\right)=\sum_{i=1}^{B}-\ln (\sigma)-\frac{n_{i}}{\sigma} .
$$

Taking partial derivatives and substituting into (27) yields $\boldsymbol{\Omega}=\left(1 / \sigma^{2}\right) \mathbf{1}_{B \times B}$, which is a rank-one matrix. We found numerically that the Fisher matrix is singular with this model. This agrees with the statement in [2] that the chosen pdf must have a local maximum. Consider instead the assumption of a "half-Gaussian" distribution (i.e., the absolute-value of a Gaussian random variable),

$$
p_{N}(\mathbf{n})=\prod_{i=1}^{B} \frac{2}{\sqrt{2 \pi} \sigma} e^{-n_{i}^{2} / 2 \sigma^{2}}, \quad n_{i} \geq 0
$$

For each individual random variable, we have the moments [16] $E\left\{n_{i}\right\}=\sqrt{2 / \pi} \sigma$ and $E\left\{n_{i}^{2}\right\}=\sigma^{2}$. The $i$ th partial derivative is

$$
\frac{\partial}{\partial n_{i}}\left(\sum_{i=1}^{B} \ln \left(\frac{2}{\sqrt{2 \pi} \sigma}\right)-\frac{n_{i}^{2}}{2 \sigma^{2}}\right)=-\frac{n_{i}}{\sigma^{2}}
$$

Taking the expectation,

$$
\begin{gathered}
E\left\{\frac{\partial}{\partial n_{i}} \ln (p(N)) \frac{\partial}{\partial n_{j}} \ln (p(N))\right\} \\
= \begin{cases}\frac{E\left\{n_{i} n_{j}\right\}}{\sigma^{4}}=\frac{2}{\pi \sigma^{2}} & i \neq j, \\
\frac{E\left\{n_{i}^{2}\right\}}{\sigma^{4}}=\frac{1}{\sigma^{2}} & i=j .\end{cases}
\end{gathered}
$$

Putting this together,

$$
\mathbf{\Omega}=\frac{1}{\sigma^{2}}\left(\frac{2}{\pi} \mathbf{1}_{B \times B}+\left(1-\frac{2}{\pi}\right) \mathbf{I}_{B}\right)
$$

which is full-rank by inspection. Hence, the half-Gaussian is a reasonable choice for an assumed density function for the NLOS phenomenon.
To extend these NLOS results to the TDOA system, we merely substitute $\overline{\mathbf{H}}$ into (26), where the matrix dimensions labeled " 2 " are increased to " 3 ."

Throughout this paper we assume a narrowband signal model, allowing us to consider the NLOS statistics to be independent of bandwidth.If the transmitted frequency were allowed to vary widely, it could significantly alter the attenuation through various materials in the environment and thereby increase the probability that the straight-line path is detectable. For the bandwidths of interest in this paper $(<100 \mathrm{MHz})$, we can safely neglect this phenomenon.

\section{GEOLOCATION FOR ROUND-TRIP TOA SYSTEMS}

Recently, a third type of time-based geolocation has been receiving renewed interest, namely, the round trip time-ofarrival (RT-TOA) system $[17,18]$. The RT-TOA approach is potentially a cost-effective solution, as none of system clocks are required to be synchronized: only short-term accuracy is needed. The basic concept entails a ranging waveform transmitted from node A to node B, which in turn sends it back to A. Node A measures the elapsed time between its transmission and reception and divides by two to determine the range. However, as quantified below, the fact that the wireless channel must be used twice will reduce the location accuracy. It should also be evident that the originating A node may be either the mobile user, or the stationary infrastructure, without changing the relevant formulas.

Consider first the round-trip ranging bound under LOS conditions. Node A will observe the elapsed round-trip time, $T_{i}=2 \tau_{i}$. Setting up the ranging bound beginning with (6) but using $s\left(t-2 \tau_{i}\right)$, and taking the derivative with respect to $\tau_{i}$, and further assuming no changes in the bandwidth or SNR conditions, we find

$$
\sigma_{\mathrm{RT}}=\frac{1}{2} \sigma_{\text {range }}
$$

where $\sigma_{\text {range }}$ was defined in (11). The fact that the rms range error is reduced by a factor of two in a round-trip geometry is well known in classical radar theory [19].

In the RT-TOA system, the B node is not a passive reflector, but receives and actively retransmits the ranging waveform. Generally, radio systems cannot receive and transmit on the same frequency at the same time without overloading the receiver's front-end. Therefore, the radio system must divide its resources into forward and reverse links. In one type of RT implementation, frequency-division (FD) multiplexing is used to separate forward and reverse links. Here, half the bandwidth is employed in the forward direction, and half in the reverse. In the FD system, the B node is particularly simple to implement; it is simply a dual-frequency transponder, although it may also add some signaling to identify the particular node returning the signal. Alternatively, time division (TD) multiplexing may be used to separate the links. Here, only half the time (hence, half the SNR) is available for the A node to measure ranging delay, and further, any synchronization error at the B node will contribute a source of ranging error. We study both FD and TD variants below. 
Consider an RT-TOA-FD system under LOS conditions. The parameter vector is $\boldsymbol{\theta}=[x, y]$, and substituting $s\left(t-2 \tau_{i}\right)$ into (13) we have,

$$
\mathbf{J}_{\theta}=\sum_{i=1}^{B}\left(\frac{1}{4} \frac{2 E}{N_{o}}\right)(2 \pi)^{2} \frac{\left|a_{i}\right|^{2}}{c^{2}}\left(\frac{\beta}{2}\right)^{2}\left(2 \mathbf{h}_{i}\right)\left(2 \mathbf{h}_{i}\right)^{H}=\frac{\mu_{1}}{4} \mathbf{H} \mathbf{\Lambda} \mathbf{H}^{H} .
$$

We observe that this differs from (15) only by several scale factors, and we account for these as follows. The RT geometry causes the factor of two that multiplies the $\mathbf{h}_{i}$ vector, just as in (34). The fact that the FD signal occupies half the bandwidth accounts for the scale factor on $\beta$. Last, the SNR is scaled by a factor of four, and this is justified as follows. To keep the total transmit power the same as in the TOA case, we assume that each node sends half the power of a TOA node. Further, the noise power contributes twice, as noise is received at nodes $\mathrm{A}$ and $\mathrm{B}$. Note that (35) leads to an rms error of exactly twice that of the TOA case.

For the RT-TOA-FD system on NLOS channels, we have that $\boldsymbol{\theta}=\left[x, y, N_{1}, \ldots, N_{B}\right]$ and $T_{i}=$ (1/ c) $\left(2 \sqrt{\left(x_{i}-x\right)^{2}+\left(y_{i}-y\right)^{2}}+2 N_{i}\right)$, where the excess NLOS distance is traveled twice. Assuming $N_{i}$ is half-Gaussian distributed, then analogous to (26), we have

$$
\begin{aligned}
\mathbf{J}_{\theta} & =\sum_{i=1}^{B}\left(\frac{1}{4} \frac{2 E}{N_{o}}\right)(2 \pi)^{2} \frac{\left|a_{i}\right|^{2}}{c^{2}}\left(\frac{\beta}{2}\right)^{2}\left[\begin{array}{c}
2 \mathbf{h}_{i} \\
2 \mathbf{e}_{i}
\end{array}\right]\left[\begin{array}{c}
2 \mathbf{h}_{i} \\
2 \mathbf{e}_{i}
\end{array}\right]^{H}, \\
\mathbf{J} & =\frac{\mu_{1}}{4}\left[\begin{array}{cc}
\mathbf{H} \boldsymbol{\Lambda} \mathbf{H}^{H} & -\mathbf{H} \boldsymbol{\Lambda} \\
-\boldsymbol{\Lambda} \mathbf{H}^{H} & \boldsymbol{\Lambda}
\end{array}\right]+\left[\begin{array}{cc}
0 & 0 \\
0 & \mathbf{\Omega}
\end{array}\right],
\end{aligned}
$$

where $\boldsymbol{\Omega}$ is defined in (33).

Consider next the RT-TOA-TD system under LOS conditions. The parameter vector is $\boldsymbol{\theta}=\left[x, y, v_{1}, \ldots, v_{B}\right]$, where $v_{i}$ denotes a synchronization error (in meters) due to B node's receiver. Here $T_{i}=(1 / c)\left(2 \sqrt{\left(x_{i}-x\right)^{2}+\left(y_{i}-y\right)^{2}}+v_{i}\right)$. As we saw in Section 3, the pdf of $v_{i}$ must be known for the CRLB to be defined. If the $\mathrm{B}$ node performs maximum likelihood delay estimation, then the synchronization error is asymptotically Gaussian distributed:

$$
\begin{aligned}
p\left(v_{i}\right) & =\frac{1}{\sqrt{2 \pi} \sigma_{\mathrm{TD}, i}} e^{-\left|v_{i}\right|^{2} / 2 \sigma_{\mathrm{TD}, i}^{2}}, \\
\sigma_{\mathrm{TD}, i} & =\frac{c}{(2 \pi) \beta \sqrt{\mathrm{SNR}_{i} / 2}} .
\end{aligned}
$$

Here $\sigma_{\mathrm{TD}}$ follows from the ranging bound (11), but the SNR has been reduced by a factor of 4 due to the RT amplifier scaling and half the observation period being available in a TD system. Then, analogous to (26), we have

$$
\begin{aligned}
& \mathbf{J}_{\theta}=\sum_{i=1}^{B}\left(\frac{1}{4} \frac{2 E}{N_{0}}\right)(2 \pi)^{2} \frac{\left|a_{i}\right|^{2}}{c^{2}} \beta^{2}\left[\begin{array}{c}
2 \mathbf{h}_{i} \\
-\mathbf{e}_{i}
\end{array}\right]\left[\begin{array}{c}
2 \mathbf{h}_{i} \\
-\mathbf{e}_{i}
\end{array}\right]^{H}, \\
& \mathbf{J}=\frac{\mu_{i}}{4}\left[\begin{array}{cc}
4 \mathbf{H} \boldsymbol{\Lambda} \mathbf{H}^{H} & -2 \mathbf{H} \boldsymbol{\Lambda} \\
-2 \boldsymbol{\Lambda} \mathbf{H}^{H} & \boldsymbol{\Lambda}
\end{array}\right]+\left[\begin{array}{cc}
\mathbf{0} & \mathbf{0} \\
\mathbf{0} & \overline{\mathbf{\Omega}}
\end{array}\right],
\end{aligned}
$$

where $\overline{\boldsymbol{\Omega}} \triangleq \operatorname{diag}\left(1 / \sigma_{\mathrm{TD}, 1}^{2}, \ldots, 1 / \sigma_{\mathrm{TD}, B}^{2}\right)$. Note that in the TD system, the signal is regenerated at node $\mathrm{B}$ and therefore there is no doubling of the noise power at node $\mathrm{A}$.
Finally, we consider the RT-TOA-TD in NLOS channels. Let $\boldsymbol{\theta}=\left[x, y, v_{1}, \ldots, v_{B}, N_{1}, \ldots, N_{B}\right]$, and $T_{i}=(1 /$ c) $\left(2 \sqrt{\left(x_{i}-x\right)^{2}+\left(y_{i}-y\right)^{2}}+v_{i}+2 N_{i}\right)$. The Fisher matrix is

$$
\mathbf{J}=\mu_{1}\left[\begin{array}{c}
2 \mathbf{H} \\
-\mathbf{I} \\
-2 \mathbf{I}
\end{array}\right]\left[\begin{array}{ll}
\frac{1}{4} & \boldsymbol{\Lambda}
\end{array}\right]\left[\begin{array}{lll}
2 \mathbf{H}^{H} & -\mathbf{I} & -2 \mathbf{I}
\end{array}\right]+\left[\begin{array}{ccc}
\mathbf{0} & \mathbf{0} & \mathbf{0} \\
\mathbf{0} & \overline{\mathbf{\Omega}} & \mathbf{0} \\
\mathbf{0} & \mathbf{0} & \mathbf{\Omega}
\end{array}\right] \text {. }
$$

\section{GEOLOCATION WITH UNCERTAIN SENSOR POSITION}

The geolocation system will consist of the mobile user and a sensor array. The positions of the sensor array have up to now been considered as fixed and known, and the user's coordinates are determined in the same frame of reference. Similarly, the GPS satellites are assumed to be at known positions, and the user's position is determined relative to them in Earth-centered coordinates. In fact, there are small errors in the satellite positions, and these must contribute to the overall GPS error budget. Imagine that we have a groundbased portable infrastructure, where the sensors are brought to a particular location, and it is desired to learn the position of various users in the immediate area. Since the system is set up quickly, the sensor positions cannot be surveyed, they are more likely to be determined via GPS. This motivates the development of the CRLB under the conditions where the sensors positions are partially known; we have a priori knowledge of the pdf from our knowledge of the GPS system error. We allow different pdfs in the various spatial dimensions, anticipating that GPS typically has poorer error performance in the $z$-direction (height). Our solution assumes that the pdfs are Gaussian, zero mean, with differing variances that are assumed known. These bounds can be developed in combination with any of the scenarios presented in previous sections, however, here we concentrate on the case of TDOA and all NLOS channels.

Let the parameter vector be

$$
\boldsymbol{\theta}=\left[x, y, \Delta_{0}, e_{x, 1}, \ldots, e_{x, B}, e_{y, 1}, \ldots, e_{y, B}, N_{1}, \ldots, N_{B}\right]^{T}
$$

of length $3+3 B$, where $e_{x, i}$ denotes the position error of the $i$ th sensor in the $x$ direction, and as before $\Delta_{0}$ is the user's clock bias, and $N_{i}$ is the $i$ th sensor's NLOS error. The $i$ th delay is

$$
\tau_{i}=\frac{1}{c}\left(\sqrt{\left(x_{i}+e_{x, i}-x\right)^{2}+\left(y_{i}+e_{y, i}-y\right)^{2}}+\Delta_{0}+N_{i}\right) .
$$

The solution is

$$
\begin{aligned}
\mathbf{J}= & \mu_{1}\left[\begin{array}{c}
\overline{\mathbf{H}}_{e} \\
\mathbf{D}_{x} \\
\mathbf{D}_{y} \\
-\mathbf{I}
\end{array}\right] \boldsymbol{\Lambda}\left[\begin{array}{llll}
\overline{\mathbf{H}}_{e}^{H} & \mathbf{D}_{x}^{H} & \mathbf{D}_{y}^{H} & -\mathbf{I}
\end{array}\right] \\
& +\left[\begin{array}{cccc}
\mathbf{0}_{3 \times 3} & & & 0 \\
& \sigma_{x}^{-2} \mathbf{I}_{B} & & \\
& & \sigma_{y}^{-2} \mathbf{I}_{B} & \\
0 & & & \mathbf{\Omega}
\end{array}\right],
\end{aligned}
$$


where

$$
\begin{aligned}
& \overline{\mathbf{h}}_{e, i}=\left[\begin{array}{c}
\frac{\left(x_{i}+e_{x, i}-x\right)}{\sqrt{\left(x_{i}+e_{x, i}-x\right)^{2}+\left(y_{i}+e_{y, i}-y\right)^{2}}} \\
\frac{\left(y_{i}+e_{y, i}-y\right)}{\sqrt{\left(x_{i}+e_{x, i}-x\right)^{2}+\left(y_{i}+e_{y, i}-y\right)^{2}}} \\
1
\end{array}\right], \\
& \overline{\mathbf{H}}_{e}=\left[\overline{\mathbf{h}}_{e, 1}, \overline{\mathbf{h}}_{e, 2}, \ldots, \overline{\mathbf{h}}_{e, B}\right] \cong \overline{\mathbf{H}} \\
& \mathbf{D}_{x}=\operatorname{diag}\left(\frac{\left(x_{1}+e_{x, 1}-x\right)}{\sqrt{\left(x_{1}+e_{x, 1}-x\right)^{2}+\left(y_{1}+e_{y, 1}-y\right)^{2}}}, \ldots,\right. \\
& \left.\frac{\left(x_{B}+e_{x, B}-x\right)}{\sqrt{\left(x_{B}+e_{x, B}-x\right)^{2}+\left(y_{B}+e_{y, B}-y\right)^{2}}}\right) \text {. } \\
& \cong \operatorname{diag}(\mathbf{H}(:, 1)) \text {. }
\end{aligned}
$$

Unlike the previous cases, here we must generate random variables $e_{x, i}, e_{y, i}$ and average over them. However, as long as these errors are small in the sense that $E\left\{\left|e_{x, i}\right|\right\} \ll E\left\{\left|x_{i}-x\right|\right\}$, their impact on the geometry term $\overline{\mathbf{H}}_{e}$ is small, and there is no need to actually generate such variables. Nevertheless, we have included them in our numerical results.

\section{NUMERICAL RESULTS}

In our initial results, we assume four sensors are placed at the corners of a square $(0,0),(0,50),(50,0),(50,50)$, and a fifth at $(25,0)$. The user location is fixed at $(15,15)$, and we assume that all links are received at the same SNR $(20 \mathrm{~dB})$, and interference is neglected. We run multiple trials of the CRLB where independent Rayleigh fading is present on each link. In Figure 1, we plot the CRLB for the TOA and TDOA systems with 2 through 5 sensors, in LOS channels. In this 2D problem, 3 sensors are the minimum required for TDOA. We note that substantial improvement is achieved with 4 sensors, and diminishing returns are setting in with a 5 th sensor. There is a noticeable loss for using TDOA versus TOA (about a 20\% increase in error). Note also that a TOA system with $B$ nodes is almost equal to a TDOA system with $B+1$.

In Figure 2, we fix the number of sensors at 4 , and we compare TOA, TDOA, and RT-TOA-FD on LOS channels. To see the sensitivity with user location, we place the user at either Location $1(20,25)$ or Location $2(10,5)$. We first note that RT-TOA-FD has a significant penalty relative to the others, with an error precisely double that of TOA. We can also see that TDOA shows somewhat more sensitivity to user location than the other two.

In the remaining figures, the user geometry more accurately models a typical indoor scenario. A square building with length $50 \mathrm{~m}$ per side is assumed, and the user location is uniformly distributed throughout. The sensors are placed on a circle surrounding the building with radius $100 \mathrm{~m}$, uniformly spaced along an arc subtending $\pm \theta$ degrees. A standard pathloss model is adopted with pathloss exponent equal to 2 (similar results were seen with 4 ), and link budget set so that all sensors experience average $\mathrm{SNR}=20 \mathrm{~dB}$ when the user is at the center of the circle. In Figure 3, we compare LOS and NLOS channels, where the NLOS is "half-Gaussian"

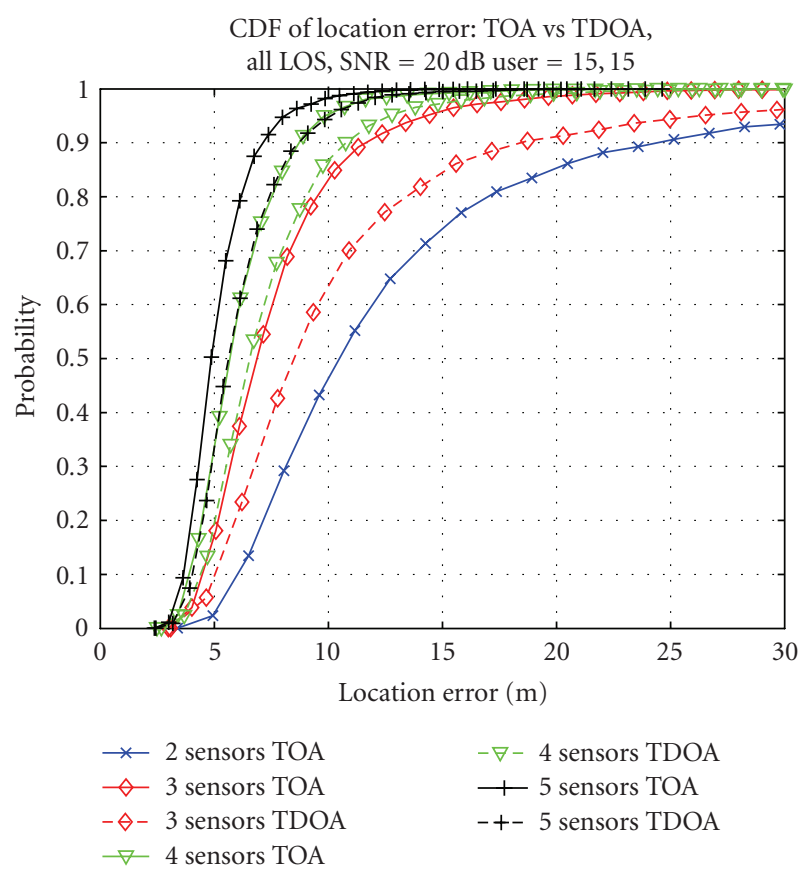

Figure 1: LOS channels, TOA versus TDOA (fixed user location).

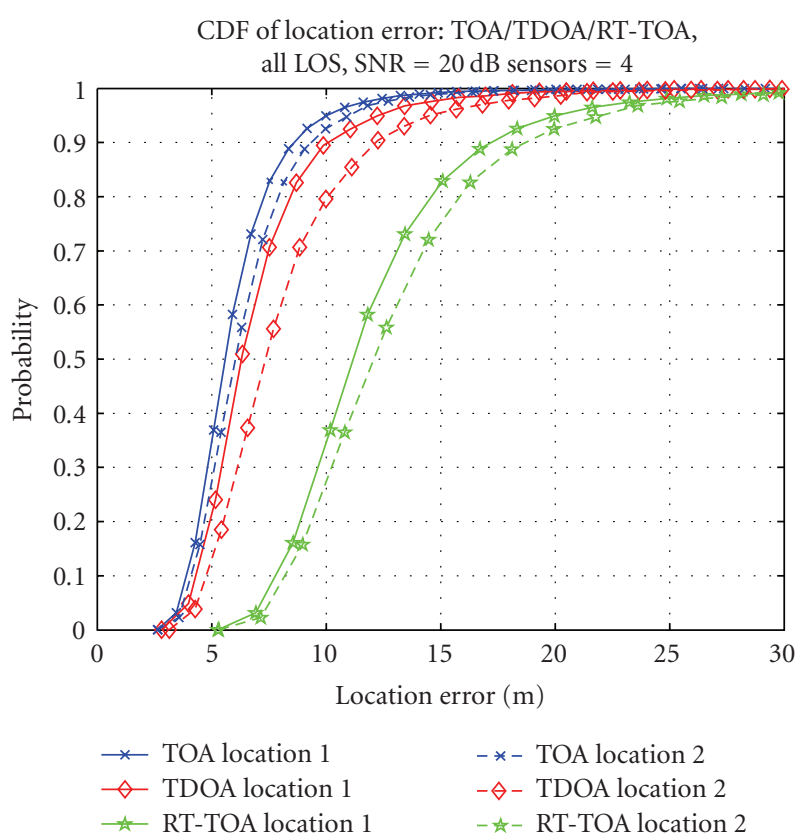

FIgURE 2: LOS channels, TOA versus TDOA versus RT-TOA-FD (two user locations).

distributed with mean $=2.5 \mathrm{~m}$. There are 4 sensors extending over half the circle $(\theta=\pi / 2)$. We compare three systems having bandwidths $1 \mathrm{MHz}, 10 \mathrm{MHz}$, and $50 \mathrm{MHz}$. For the $1 \mathrm{MHz}$ system (similar to today's civilian GPS), we see a location error on the order of $10 \mathrm{~m}$, and at $90 \%$ there is a loss of about $2 \mathrm{~m}$ (roughly 20\%) in the NLOS case. When the system bandwidth is increased to $10 \mathrm{MHz}$, the LOS case shows a 10-fold improvement in the location error (as expected), 


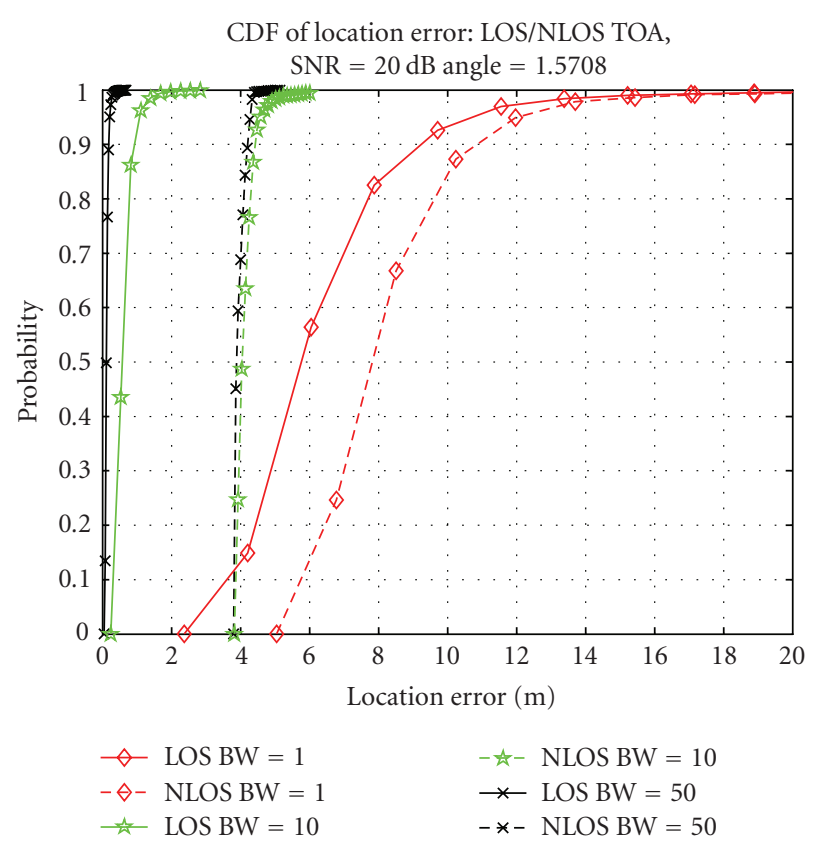

FIgUre 3: TOA channels, LOS versus NLOS (three bandwidths).

but the NLOS shows only a 2 -fold improvement. When the bandwidth is further increased to $50 \mathrm{MHz}$, the NLOS channel shows almost no improvement. Clearly, arbitrary increases in the signaling bandwidth are not warranted under NLOS conditions.

Figure 4(a) is for the $1 \mathrm{MHz}$, TDOA system, where sensor location error is included (with standard deviation $2.5 \mathrm{~m}$ ). In this plot, the spacing between the 4 sensors is varied so that the arc subtended corresponds to $\theta=\{\pi / 4,3 \pi / 8, \pi / 2\}$. We see that spacing the sensors at $\pm \pi / 4$ incurs a large loss, although $\pm 3 \pi / 8$ appears to be acceptable. For good performance with TDOA, the sensors should span almost a half circle around the building. In Figure 4(b), we repeat the above conditions for a $10 \mathrm{MHz}$ system, and we see again that the LOS case gets a 10 -fold improvement, and the NLOS case exhibiting diminishing returns as before. Note also that the combination of NLOS channels and narrow angular spacing gives particularly poor results.

Figure 5 (bandwidth $=10 \mathrm{MHz}$ ) is a comparison of TOA, TDOA, and RT-TOA-FD for LOS channels, where we have varied the sensor position angle. This plot highlights the sensitivity of TDOA to geometry factors (as anticipated in Figure 2) leading to a change in the relative ordering among the techniques. For relatively large angles, such as $\pm \pi / 2$, TDOA is better than RT-TOA-FD; for $\pm 3 \pi / 8$, they are quite close; and for small angles such as $\pm \pi / 4$, TDOA is significantly worse than RT-TOA-FD. The choice among these modes depends on how the system sensors will be deployed.

Figure 6 concentrates on the RT-TOA system in $10 \mathrm{MHz}$, with sensors covering $\pm \pi / 2$. The left-most group consists of four curves for LOS channels. We plot the RT-TOA-FD and $\mathrm{TD}$, along with the TOA and TDOA curves for reference. We observe that TD is superior to FD and comes quite close to TOA. The NLOS curve is intermediate, and would shift con-

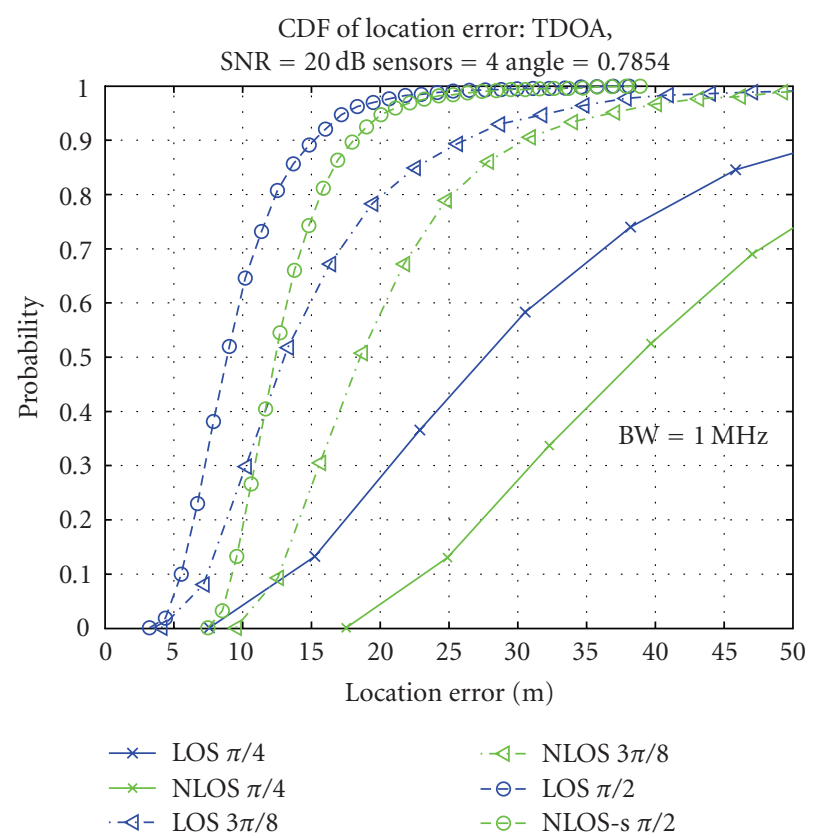

(a) TDOA with sensor error, LOS versus NLOS (variable sensor position, $1 \mathrm{MHz}$ ).

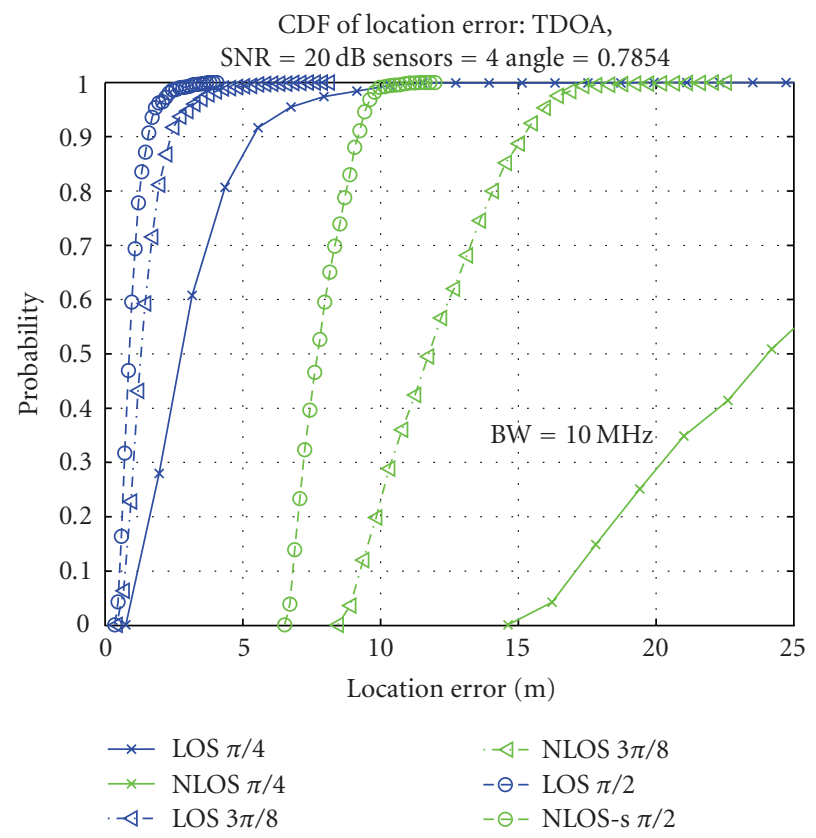

(b) TDOA with sensor error, LOS versus NLOS (variable sensor position, $10 \mathrm{MHz}$ ).

FIGURE 4

siderably to the right if the sensor angle were made smaller. For the four NLOS cases, TD is again superior to FD, and quite close to TOA. TDOA is dramatically worse, as was also seen in Figure 4(b). It appears that RT-TOA can give significant benefit over TDOA, depending on the sensor angle and channel conditions. 


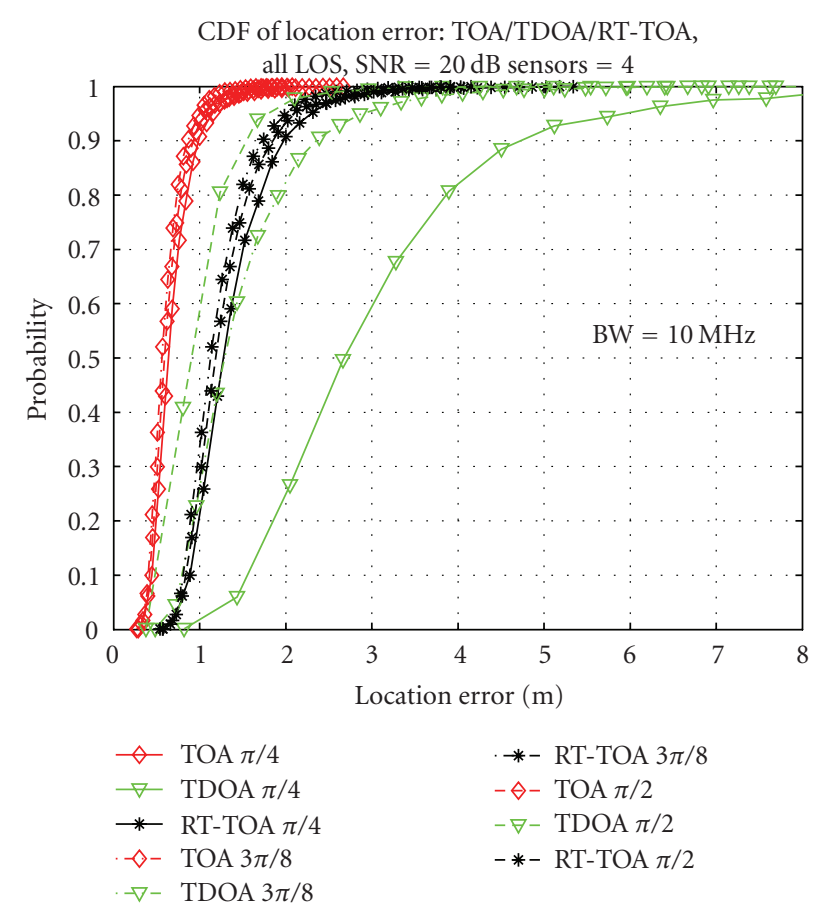

FIgUre 5: LOS channels, TOA versus TDOA versus RT-TOA-FD (3 angles).

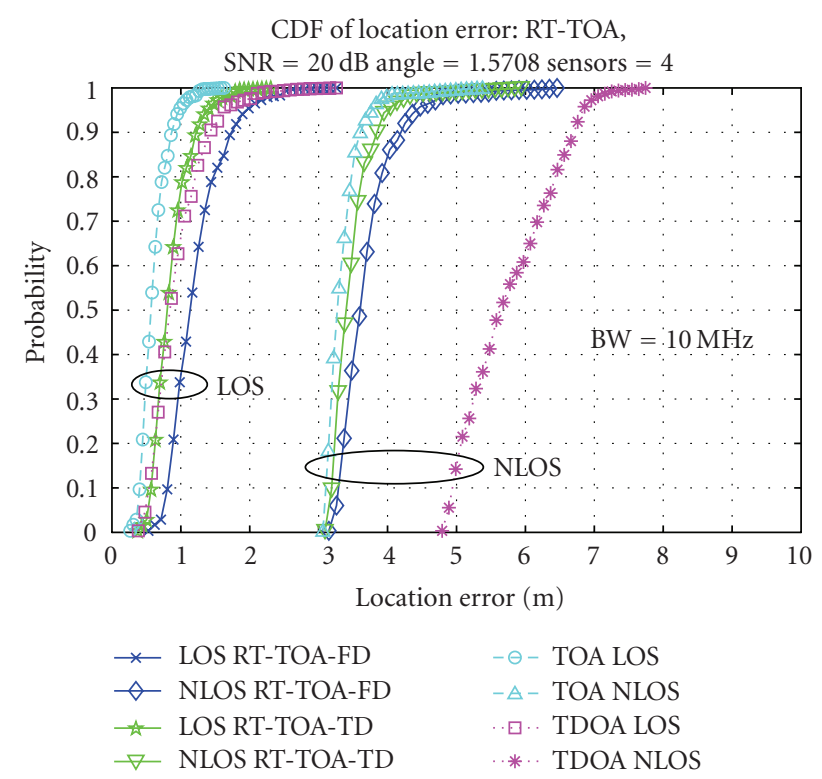

FIgURE 6: RT-TOA in LOS and NLOS $(2.5 \mathrm{~m})$ channels: FD versus TD.

\section{SUMMARY AND CONCLUSIONS}

We have investigated the Cramer-Rao bounds for three timebased geolocation schemes, in LOS and all-NLOS channel conditions. Under pure NLOS conditions, without a priori information, we proved that the Cramer-Rao bound does not exist. We introduced a half-Gaussian model for NLOS with positive-only support and found that the related bound ex- ists. On the other hand, lack of a priori amplitude information was found not to affect the bound. While increasing the bandwidth in LOS channels leads to a proportional increase in location accuracy, we find that in NLOS channels bandwidth increase beyond a certain point $(10 \mathrm{MHz}$ in our examples) does not lead to significant gains. We introduced a new bound for round-trip time-of-arrival systems, in which twoway signaling is used and accurate clocks are not required. Among the time-based location techniques, TOA was found to have the best performance, and RT-TOA-FD has exactly twice the location error of TOA in LOS channels. The relative ordering between TDOA and RT-TOA was found to depend on the sensor geometry, with TDOA being preferable if the sensors cover approximately half a circle or more around the user. RT-TOA-TD is slightly better than FD, and comes quite close to TOA in either LOS or NLOS channels.

Future research is required to determine similar bounds under multipath conditions, and most importantly, to find practical algorithms that can approach the performance levels promised by the bounds.

\section{REFERENCES}

[1] S. Gezici, Z. Tian, G. B. Giannakis, et al., "Localization via ultra-wideband radios: a look at positioning aspects of future sensor networks," IEEE Signal Processing Magazine, vol. 22, no. 4, pp. 70-84, 2005.

[2] Y. Qi, H. Kobayashi, and H. Suda, "Analysis of wireless geolocation in a non-line-of-sight environment," IEEE Transactions on Wireless Communications, vol. 5, no. 3, pp. 672-681, 2006.

[3] Y. Qi, H. Suda, and H. Kobayashi, "On time-of-arrival positioning in a multipath environment," in Proceedings of the 60th IEEE Vehicular Technology Conference (VTC '04), vol. 5, pp. 3540-3544, Los Angeles, Calif, USA, September 2004.

[4] Y. Qi and H. Kobayashi, "Optimum positioning receiver for non-synchronized mobile systems," in Proceedings of the 37th Annual Conference on Information Sciences and Systems (CISS '03), Baltimore, Md, USA, March 2003.

[5] M. P. Wylie and J. Holtzman, "The non-line of sight problem in mobile location estimation," in Proceedings of the 5th IEEE International Conference on Universal Personal Communications (ICUPC '96), vol. 2, pp. 827-831, Cambridge, Mass, USA, September-October 1996.

[6] P.-C. Chen, "A non-line-of-sight error mitigation algorithm in location estimation," in Proceedings of IEEE Wireless Communications and Networking Conference (WCNC '99), pp. 316320, New Orleans, La, USA, September 1999.

[7] S. Al-Jazzar, J. Caffery Jr., and H.-R. You, "A scattering model based approach to NLOS mitigation in TOA location systems," in Proceedings of the 55th IEEE Vehicular Technology Conference (VTC '02), vol. 2, pp. 861-865, Birmingham, Ala, USA, May 2002.

[8] X. Wang, Z. Wang, and B. O’Dea, “A TOA-based location algorithm reducing the errors due to non-line-of-sight (NLOS) propagation," IEEE Transactions on Vehicular Technology, vol. 52, no. 1, pp. 112-116, 2003.

[9] B. Alavi and K. Pahlavan, "Analysis of undetected direct path in time of arrival based UWB indoor geolocation," in Proceedings of the 62nd IEEE Vehicular Technology Conference (VTC '05), vol. 4, pp. 2627-2631, Dallas, Tex, USA, September 2005 . 
[10] C. Gentile and A. Kik, "An evaluation of ultra wideband technology for indoor ranging," in Proceedings of IEEE Global Telecommunications Conference (GLOBECOM '06), pp. 1-6, San Francisco, Calif, USA, November-December 2006.

[11] E. Kaplan and C. J. Hegarty, Eds., Understanding GPS: Principles and Applications, Artech House, Norwood, Mass, USA, 2006.

[12] C. Rizos, "Network RTK research and implementation-a geodetic perspective," Journal of Global Positioning Systems, vol. 1, no. 2, pp. 144-150, 2002.

[13] L. L. Scharf, Statistical Signal Processing: Detection, Estimation, and Time Series Analysis, Addison-Wesley, Reading, Mass, USA, 1991.

[14] H. L. Van Trees, Detection, Estimation and Modulation Theory, Part 1, John Wiley \& Sons, New York, NY, USA, 1968.

[15] H. V. Poor, An Introduction to Signal Detection and Estimation, Springer, New York, NY, USA, 1988.

[16] A. Papoulis, Probability, Random Variables, and Stochastic Processes, McGraw-Hill, New York, NY, USA, 1965.

[17] D. D. McCrady, L. Doyle, H. Forstrom, T. Dempsey, and M. Martorana, "Mobile ranging using low-accuracy clocks," IEEE Transactions on Microwave Theory and Techniques, vol. 48, no. 6, pp. 951-958, 2000.

[18] S. Kim, A. P. Brown, T. Pals, R. A. Iltis, and H. Lee, "Geolocation in ad hoc networks using DS-CDMA and generalized successive interference cancellation," IEEE Journal on Selected Areas in Communications, vol. 23, no. 5, pp. 984-998, 2005.

[19] C. Cook and M. Bernfeld, Radar Signals: An Introduction to Theory and Application, Academic Press, New York, NY, USA, 1967. 